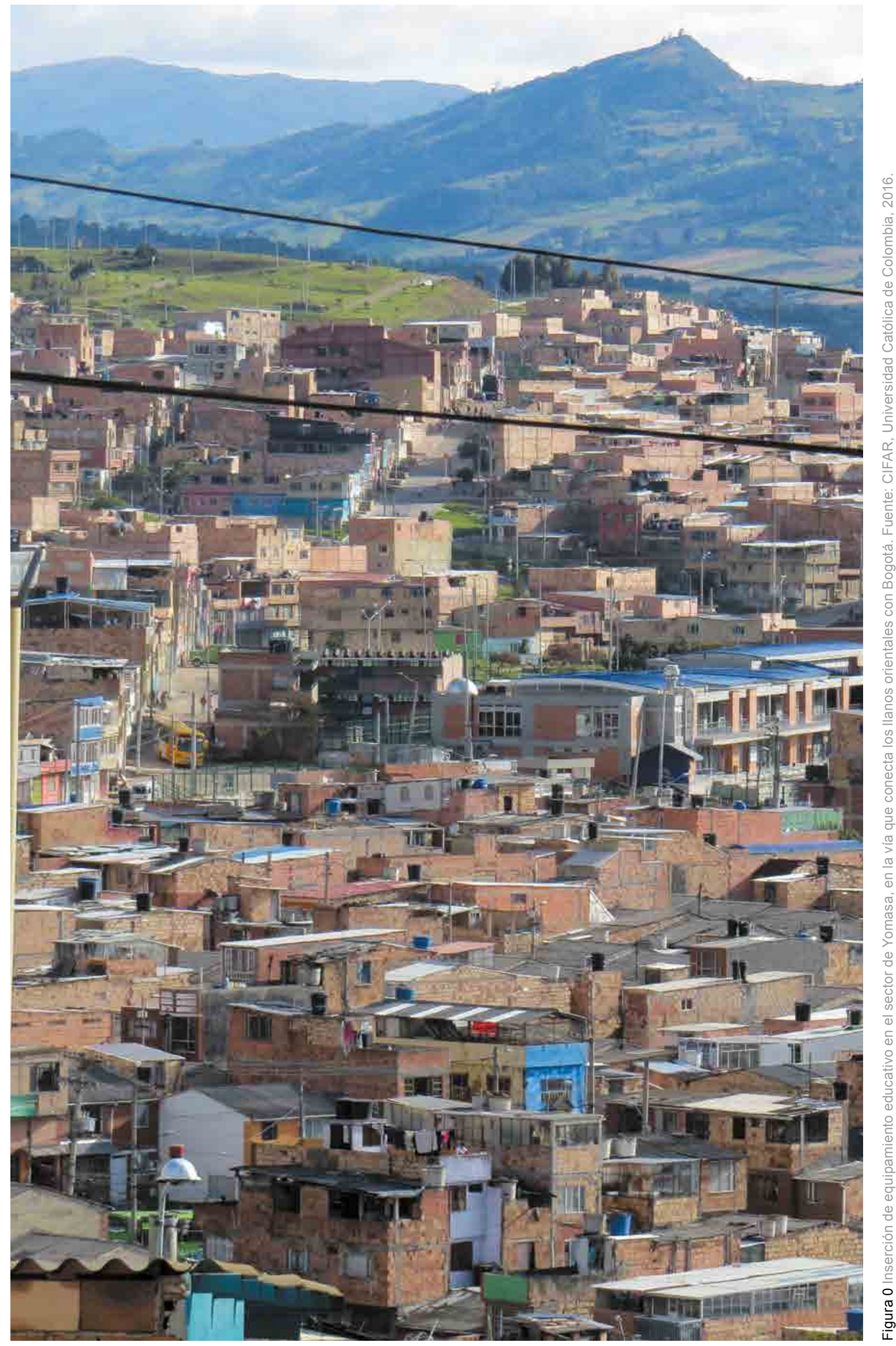



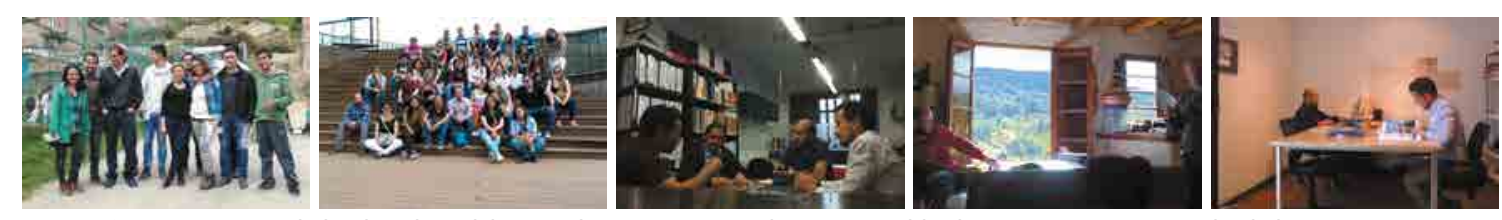

Secuencia: Distintas actividades de trabajo del Grupo de Investigación Hábitat sustentable, diseño integrativo y complejidad Fotos: Hernando Verdugo, Jairo Ovalle, Ángelo Páez

\title{
EQUIPAMIENTO URBANO EN LA RECONSTRUCCIÓN DE VÍNCULOS COMUNITARIOS'
}

\author{
URBAN EQUIPMENT IN THE RECONSTRUCTION OF COMMUNITY TIES'
}

\author{
Jairo Hernán Ovalle Garay², Ángelo Páez Calvo³
}

\section{RESUMEN}

Este texto indaga sobre el rol estratégico del equipamiento en la construcción física de la ciudad latinoamericana, a partir de su capacidad para transformar entornos urbanos no consolidados como insumo para una futura intervención en el borde suroriental de la ciudad de Bogotá. El trabajo es desarrollado en dos partes. Primero construye un marco desde la relación del equipamiento con tres temas: ciudad, participación ciudadana y espacio público. La segunda parte es un análisis a un repertorio de equipamientos de diferente escala de los últimos 60 años en la ciudad latinoamericana, para identificar constantes de implantación y relación con el entorno. Como conclusión, el artículo señala que es necesario repensar la relación del equipamiento con el espacio público a la luz de la problemáticas del suelo existentes, así como el papel del proyecto en la modelación de objetos que trascienden el hecho normativo y sugieren un camino que abandona las prácticas en el planeamiento de la ciudad ancladas en convencionalismos funcionales.

Palabras clave: expansión urbana, equipamiento urbano, asentamientos urbanos, espacio público, participación comunitaria.

\section{ABSTRACT}

This paper investigates the strategic role of equipment in the physical construction of the Latin American city, based on its capacity to transform unconsolidated urban environments, as a component for a future intervention in the southeastern edge of the city of Bogota. The text consists of two parts. First, it builds a framework that originates in the relationship of equipment with three subjects: city, citizen participation and public space. Second, it analyzes a repertoire of equipment of different scales from the last 60 years in the Latin American city, to identify constants associated with implantation and relationship with the environment. As a conclusion, this article affirms that it is necessary to rethink the relationship between equipment and public space in light of existing land problems, as well as the role of projects in the modeling of objects that transcend normative fact and suggest a path that leaves behind city planning practices anchored in functional conventions.

Keywords: urban expansion, urban equipment, urban settlements, public space, community participation.

Artículo recibido el 3 de diciembre de 2016 y aceptado el 24 de mayo de 2017 DOI: https://doi.org/10.22320/07196466.2017.35.051.05

[1] Este artículo está basado en los resultados de investigación titulada "El impacto de los equipamientos colectivos en los procesos de rehabilitación urbana - caso de estudio borde urbano de Bogotá - Gran Yomasa", código 1110016, patrocinada por la Dirección Centra de Investigaciones de la Universidad Católica de Colombia, 2016, Grupo de investigación "Hábitat sustentable, diseño integrativo y complejidad", Facultad de Diseño, Universidad Católica de Colombia.

[2] Centro de Investigaciones de la Facultad de Diseño. Universidad Católica de Colombia, Bogotá, Colombia. jhovalle@ucatolica. edu.co

[3] Centro de Investigaciones de la Facultad de Diseño. Universidad Católica de Colombia, Bogotá, Colombia. apaez@ucatolica.edu. co 


\section{La construcción del borde en América Latina}

Este texto es parte del proyecto de investigación "El impacto de los equipamientos colectivos en los procesos de rehabilitación urbana - caso de estudio borde urbano de Bogotá - Gran Yomasa", adscrito al desarrollo investigativo del grupo de investigación en "Hábitat sustentable, diseño integrativo y complejidad", de la Facultad de Diseño de la Universidad Católica de Colombia; en el marco de un proyecto institucional, que busca la articulación de las instituciones académicas en la solución de problemas en contextos reales y establece un insumo para una posible intervención junto con la comunidad en el futuro, a partir de la idea de que los equipamientos urbanos de uso colectivo son componentes primarios de la estructura de la ciudad que contribuyen al ordenamiento y planeación de la misma, desde su valor estratégico, particularmente en entornos urbanos no consolidados, donde su función, identidad y significado, resulta crucial para la efectiva articulación con el tejido urbano ${ }^{4}$.

El problema es que no siempre las condiciones de desarrollo de la ciudad están articuladas y el entorno urbano en el que se inscriben estas intervenciones es el resultado de la premura con la cual se llevan a cabo los procesos de planeación que no prevén su impacto real en los habitantes, al desconocer la totalidad de las necesidades y deja en un segundo plano el rol del equipamiento como elemento estructural y estructurante de la ciudad.

Así, pues, éste artículo tiene como objetivo profundizar en el papel de los equipamientos urbanos en la consolidación de la ciudad, vistos desde su implantación y relación con el contexto, y se ampara en la idea de que tanto la ciudad como su arquitectura deben estructurase armónicamente en aras de la óptima articulación de sus funciones y servicios, con miras al mejoramiento de las relaciones entre las comunidades. Asimismo, se debe pensar desde lo local el impacto hacia otros territorios de origen informal ubicados en los bordes urbanos de las ciudades latinoamericanas y que son parte o se encuentran en proceso de legalización, como un reconocimiento administrativo que implica la construcción posterior en el tiempo de las infraestructuras básicas para el cubrimiento de las necesidades de la población (Urrea, del Castillo y Montaña, 2011).

En este escenario, que constituye tan solo una de las miradas sobre el territorio de borde urbano, para el caso específico de Bogotá, fue seleccionada para su 


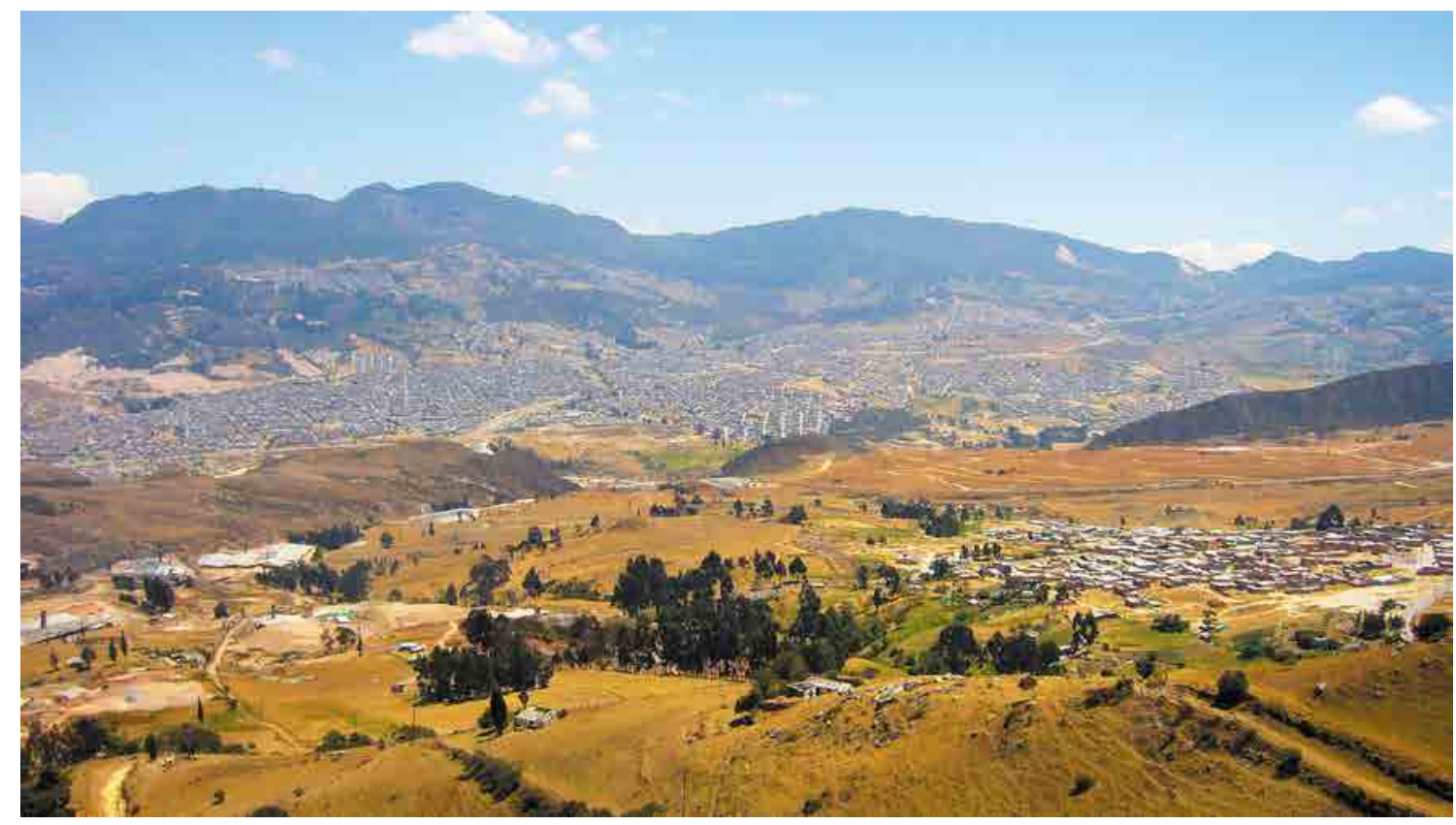

Figura 1 Vista panorámica del borde suroriental de Bogotá desde la zona montañosa de Ciudad Bolívar. En primer plano, el parque minero y el relleno sanitario de doña Juana. Al frente, el área de interés: el sector de Gran Yomasa atravesado por la autopista que conecta con la región de los llanos orientales. Fuente: Fotografía de Jairo Ovalle (2010).

estudio la zona de Yomasa (Figura 1), ubicada al suroriente del casco urbano, en un territorio que no ha sido ajeno a los procesos de crecimiento espontáneo (Alexiou, 2004), donde la expansión urbana desarrollada al margen de políticas de planeación llega a ser equivalente en extensión a la desarrollada con procesos planificados ${ }^{5} y$, en consecuencia, existen centros poblados excluidos del desarrollo urbano donde "...no se estudia, ni se planifica, ni se presupuesta por parte de la administración pública ninguna infraestructura para el transporte, los servicios públicos domiciliarios, ni la dotación de equipamientos" (Urrea, del Castillo y Montaña, 2011:81). (Figura 2) De esta forma, hay grandes extensiones de tierra con tejidos residenciales en manos de los parceladores ${ }^{6}$ y una economía que atiende la población no cubierta por las políticas públicas de vivienda, en terrenos en los cuales no hay un control administrativo de la planeación ${ }^{7}$. Y aunque esta situación ha sido reconocida como procesos informales desde la década de 1970, con diferentes proyectos como "obras con saldo pedagógico", el programa de "mejoramiento integral de barrios" o los proyectos para reubicación y mitigación del riesgo de población que habita en zonas de riesgo por remoción en masa o inundación (Rubio y Castellanos, 2006), ante los índices de crecimiento y expansión de la ciudad, constituye una tarea en permanente construcción.

Desde esa perspectiva, es necesario articular la investigación al pensamiento sistémico para reconocer la importancia de la mirada integrada de los problemas y entender el acercamiento, desde una tensión constructiva capaz de hacer evidente la simultaneidad de los actuales sistemas dinámicos y complejos establecidos en el territorio, los cuales deberían dar lugar a aproximaciones diferentes para la investigación proyectual orientada hacia la acción, la adaptación, la flexibilidad y la autorregulación (Centro de Investigaciones de la Facultad de Diseño, CIFAR, 2015).

[5] El crecimiento como expansión urbana podría clasificarse en dos tipos: 1. Crecimiento planificado (Macroproyectos o proyectos estratégicos) y, 2. Crecimiento espontáneo.

[6] El parcelador es aquel agente que "a sabiendas de que no está previsto ni presupuestado ningún servicio, ni infraestructura, toma la decisión de lotear e iniciar venta clandestina sin ninguna obra de urbanismo" (Urrea, del Castillo y Montaña, 2011: 81).

[7] Este fenómeno ocurre en casi todos los países del tercer mundo, donde un porcentaje que varía de acuerdo a cada nación, habita en barrios y centros urbanos al margen de procesos de planeación 


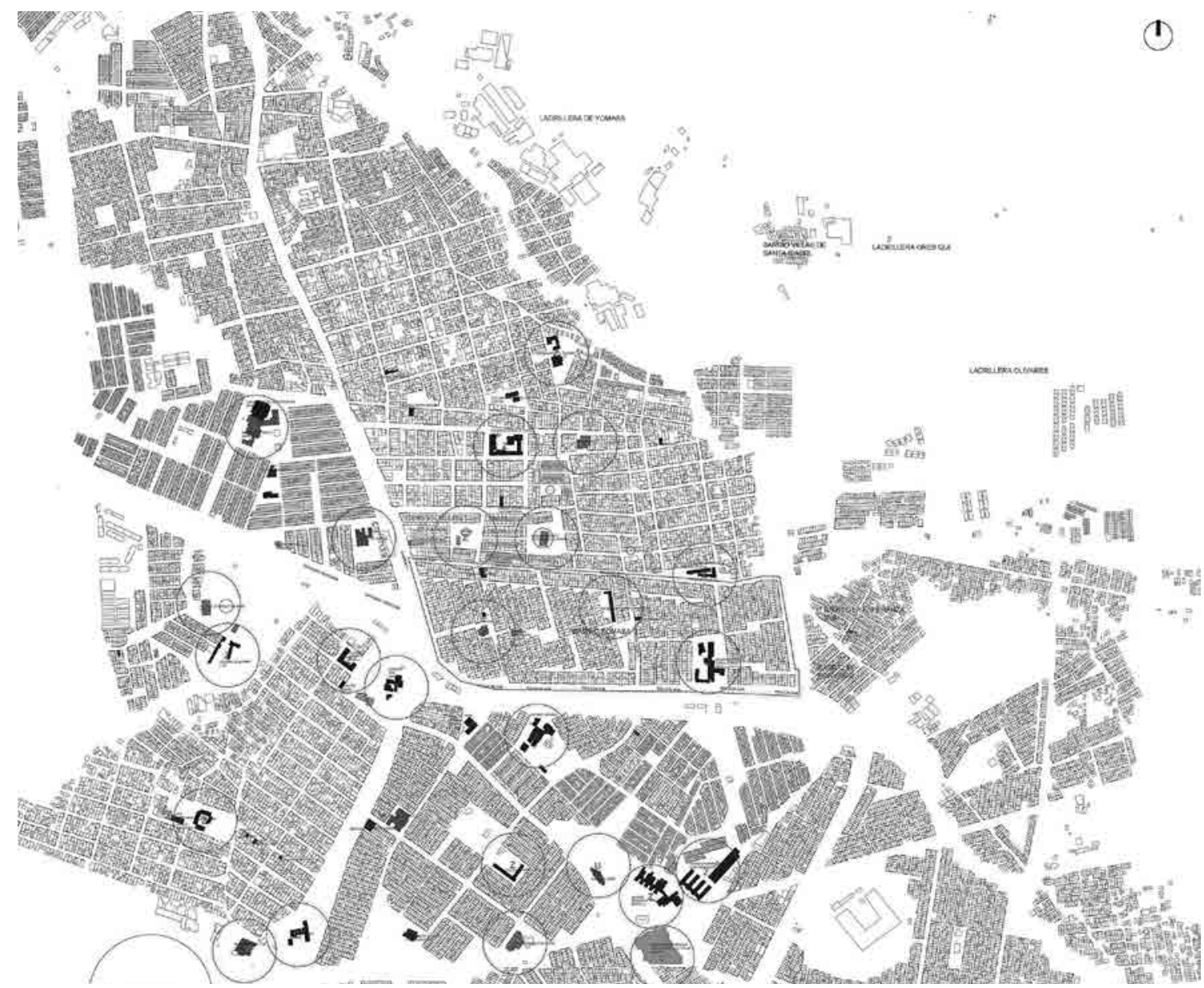

Figura 2 Plano de localización de los equipamientos existentes en el sector de Gran Yomasa. Fuente: Plano dibujado por Cristian Calderón sobre una base cartográfica digital, 2016.

\section{METODOLOGÍA}

El presente artículo está estructurado en dos partes. La primera desarrolla el papel del equipamiento en la construcción del borde urbano y su importancia para incorporar a la ciudadanía como un agente de transformación; problemática analizada desde el enfoque de varios autores. La segunda parte está enfocada en la revisión de proyectos fundamentalmente equipamientos, desde el análisis del diseño como una herramienta didáctica para encontrar constantes y puntos en común como instrumentos de intervención y desarrollo ${ }^{8}$. Para esto, la primera parte plantea la revisión de fuentes bibliográficas desde un enfoque descriptivo e identifica tres actores relevantes en la mirada sobre los equipamientos fundamentados en su relación con la construcción del territorio. El primero de ellos hace referencia al aspecto morfológico y urbano visto desde la relación con el tejido de la ciudad; el segundo aspecto se relaciona con la integración de la comunidad en los procesos de rehabilitación urbana; y el último, lo constituye el espacio público y su papel como un instrumento de disolución de límites e intermediario entre el equipamiento y sus habitantes.

Desde aquí se da forma a la revisión del repertorio de proyectos ubicados en áreas centrales y de borde en Latinoamérica, los cuales han generado un impacto reconocible en su entorno. La presentación de los proyectos es organizada en una matriz que establece una mirada simultánea sobre los aspectos más relevantes en el desarrollo de estos equipamientos en cuanto a su implantación, con el propósito de identificar los criterios de implantación y desarrollo. Tales aspectos son agrupados en tres tipos de relaciones temáticas: la relación con los aspectos naturales, la relación con el contexto construido y la relación entre lo público y lo privado. Cada una de éstas posee un componente cualitativo que establece la principal característica graficada en planta, corte o alzado.

[8] El análisis como una actividad experimental ha sido ampliamente desarrollado en la Escuela de Arquitectura de la Universidad de Delft y ha permitido construir una línea de pensamiento alrededor de la construcción de nexos entre la enseñanza y la investigación a través del estudio del proyecto. Esta investigación establece lazos con aquella línea de pensamiento y generación de conocimiento, y se plantea como una primera mirada para el desarrollo de herramientas o criterios de intervención en el borde urbano a través de los equipamientos. 
En julio de 1951, se realizó en la ciudad de Hoddesden (Inglaterra) el VIII Congreso Internacional de Arquitectura Moderna titulado "The heart of the city", el cual se enfocó en los problemas "...de aquellos sectores urbanos que son lugar de congregación de masas, centros de vida colectiva y, al mismo tiempo, símbolos de la ciudad misma" (Rogers, Sert y Tyrwhitt, 1955). Allí, se examinó el papel de la arquitectura en la construcción de las relaciones de los habitantes con sus ciudades, desde la recuperación de vínculos perdidos por conflictos armados o afectados por procesos de reconstrucción desligados de la estructura tradicional de la ciudad.

Se buscaba entonces un "corazón urbano" -llamado con posterioridad "centro cívico"-, que estableciera el punto de partida en la indagación desde la arquitectura moderna para la construcción de ciudadanía, donde su función social tuviera un fin: "...unir a la gente y facilitar los contactos directos y el intercambio de ideas que estimulen la libre discusión" (Ibídem, 8). Así, los congresos internacionales reconocían los centros tradicionales de las ciudades, desde una arquitectura basada en el equipamiento y su relación con el espacio público, como elementos esenciales para la vitalidad de dicho corazón, como un instrumento de reconstrucción de tejidos fragmentados.

\section{Equipamiento y ciudad}

Según lo anterior, hablar de los equipamientos y su relación con la ciudad es hablar del origen de la ciudad misma, porque es en ella donde se construye más y donde surgen los equipamientos de mayor dimensión y protagonismo ${ }^{9}$ (Cruz, 2007), como parte del sistema primario estructurante de la morfología entre los espacios abiertos y las construcciones (Amaya et al., 2004). Al respecto, Aldo Rossi (1971) aborda el papel estratégico de los equipamientos colectivos en la estructuración del territorio en relación con el sentido del lugar (geniuslocci), relacionados con la conglomeración de personas y la creación de símbolos que hacen legible y propio el territorio (Figura 3).

En tal sentido, loannis Alexiou considera muy importante esta idea de construcción simbólica, porque representa y materializa las mismas instituciones colectivas de cada sociedad (Amaya et al., 2004). Además, es necesario pasar de una serie de edificaciones que ofrecen servicios para satisfacer una necesidad básica, a una serie de soportes que hagan la triangulación indispensable en el equipamiento: mejorar la calidad de vida del territorio, aportar a la calidad de vida de la comunidad que los usa y fomentar la competitividad y productividad urbana (ibídem) (Figura 4).

La delimitación de la escala de aproximación de esta investigación toma en cuenta la articulación de la estructura urbana, conformada por el conjunto de elementos primarios (equipamientos, usos colectivos), y la zona residencial, las que conectadas por los elementos de circulación (calles, plazas) generan los "componentes morfológicos integrales" en diferentes escalas y determinan la estructura del tejido urbano y sus grados de interacción ${ }^{10}$.

Así, la lectura de la ciudad como un hecho complejo (Morin, 1998) es identificable como una agrupación de asentamientos con tejidos densamente establecidos, estructuras funcionales que requieren de aproximaciones especiales $y$, en la mayoría de los casos, no cuentan con un sistema de planeación que les permita ser eficientes y adecuados.

El barrio es, entonces, la "unidad básica" para la identidad de una comunidad"1" que, con algunas variaciones físico-espaciales o socioeconómicas, construye una directa relación entre los tejidos de una población y la cohesión e identidad de una comunidad como fórmula alternativa para disminuir la precariedad inicial de estos sectores.

\section{La participación ciudadana}

Frente a los usuarios, el equipamiento supera la noción de indicador de cobertura y pasa a ser un actor determinante en la planeación del territorio; $y$ aunque en éstos procesos, la participación de los ciudadanos es un principio reconocido por las legislaciones urbanísticas, "existe una sensibilidad creciente que no quiere seguir consintiendo que los poderes democráticos sigan haciendo la ciudad para la gente pero sin la gente. Porque el aparato de propaganda con el que el poder dota a sus programas, destinado a garantizar el entusiasmo por sus estrategias urbanas es, sin duda, mayor que el destinado a garantizar espacios de participación efectiva" (Vergara y De las Rivas, 2014: 134).

Entonces, ¿cómo saber que estos procesos de mejoramiento e incorporación de tejidos en la estructura de la ciudad generan un impacto en las redes y los lazos de la comunidad? En este sentido, el empoderamiento de la comunidad en torno a las decisiones sobre su entorno, trasciende el objetivo de articular zonas de la ciudad con la estructura funcional y de servicios hacia la definición de una imagen reconocible para los habitantes. Con esto, los equipamientos abordan la función asignada, como generar sentido de pertenencia (Franco y Zabala, 2012). Son estas intervenciones las que hacen "legible" el territorio, construido desde la percepción y las vivencias de sus habitantes (Lynch, 1970) (Figura 5).

En las décadas de 1970 y 1980, los efectos negativos de los grandes planes de renovación sufrieron serios cuestionamientos que abrieron la puerta hacia otras formas de intervenir y detectar las problemáticas urbanas, más próximas a los habitantes. De esa forma, el diseño urbano encontró en los procesos de recuperación, operaciones catalizadoras de transformaciones urbanas, puestas en práctica en ciudades como Barcelona, bajo el nombre de acupuntura urbana (durante los 80) o, en el caso de Brasil, en la ciudad de Curitiba, durante la alcaldía de Jaime Lerner ${ }^{12}$. Su acción sobre puntos específicos y de alto significado para sus habitantes, otorgaron la capacidad para lograr una cura a un malestar general (Casanova y Hernández, 2014) ${ }^{13}$. Este camino destaca el

[9] Ante la pregunta “¿también pasan más cosas en la ciudad desde el punto de vista arquitectónico?”, realizada por Valdemar Cruz y publicada originalmente por Campo das letras en el libro Retratos de Siza (Cruz, 2007)

[10] Los componentes morfológicos integrales son: La calle, la manzana ortogonal y sus variaciones, el barrio, el sector, la centralidad, el equipamiento especial, el sistema de movilidad (Alexiou, 2004)

[11] "La mayoría de las personas estructura su ciudad hasta, cierto punto, en esta forma, quedando margen para las diferencias individuales en cuanto a si las sendas o los barrios son los elementos preponderantes. Esto parece depender no sólo del individuo sino también de la en cuanto a si las sendas olos barrios son 


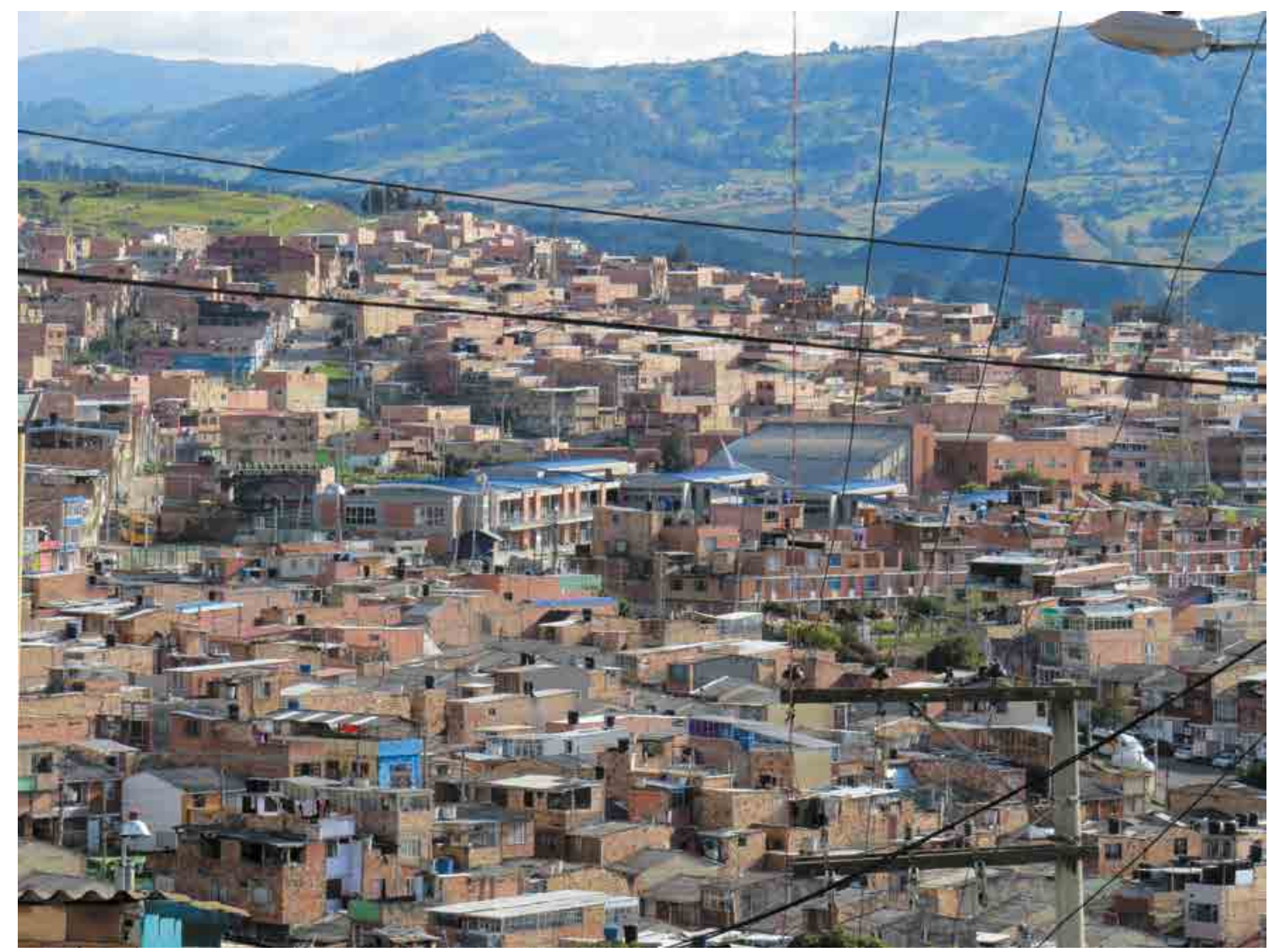

Figura 3 Inserción de equipamiento educativo en el sector de Yomasa, en la vía que conecta los llanos orientales con Bogotá. Fuente: CIFAR, Universidad Católica de Colombia, 2016.

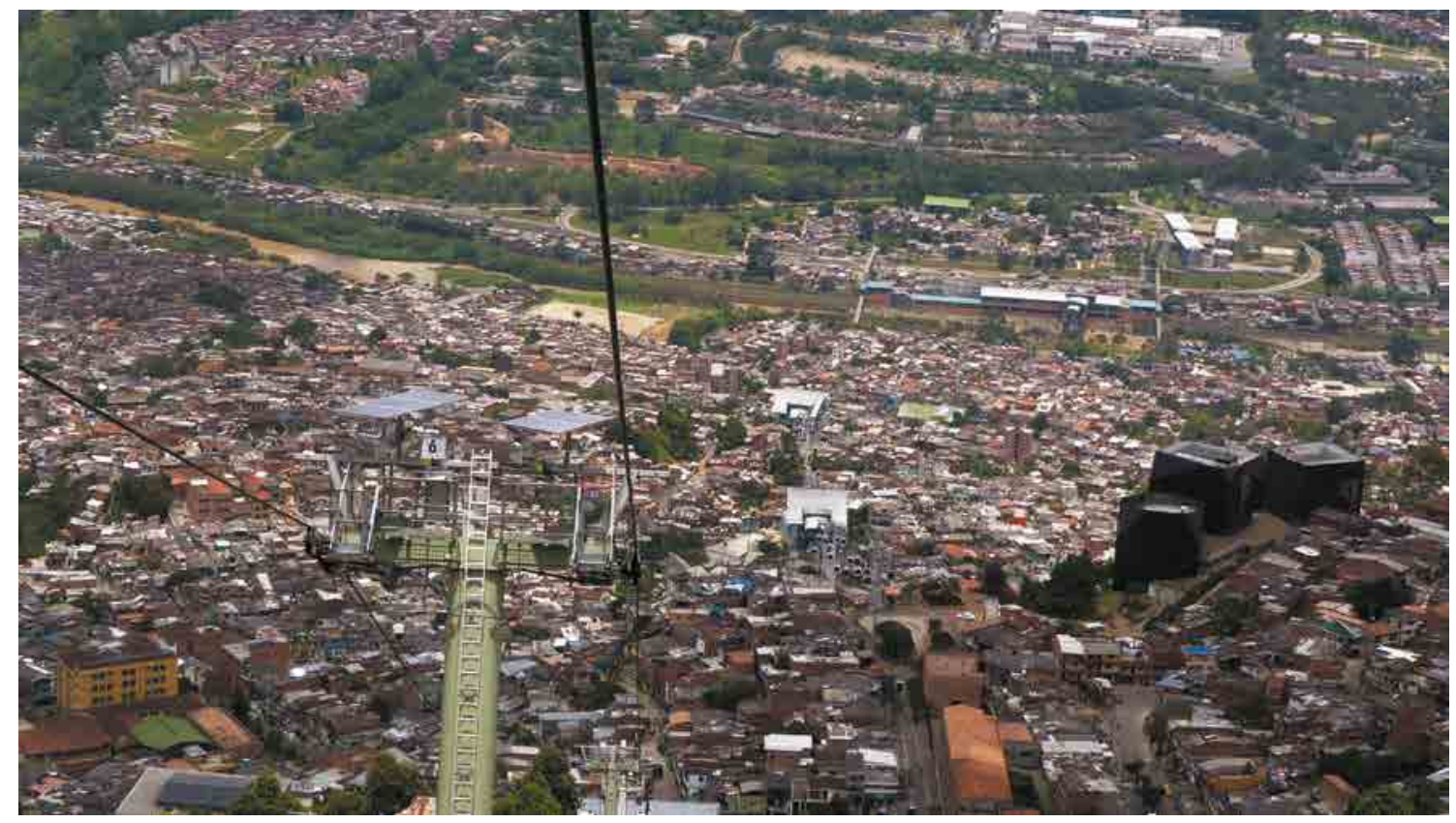

Figura 4 Estructuras urbanas en la integración de los equipamientos y tejidos residenciales (Medellín). Fuente: Fotografía de Jairo Ovalle (2012). 


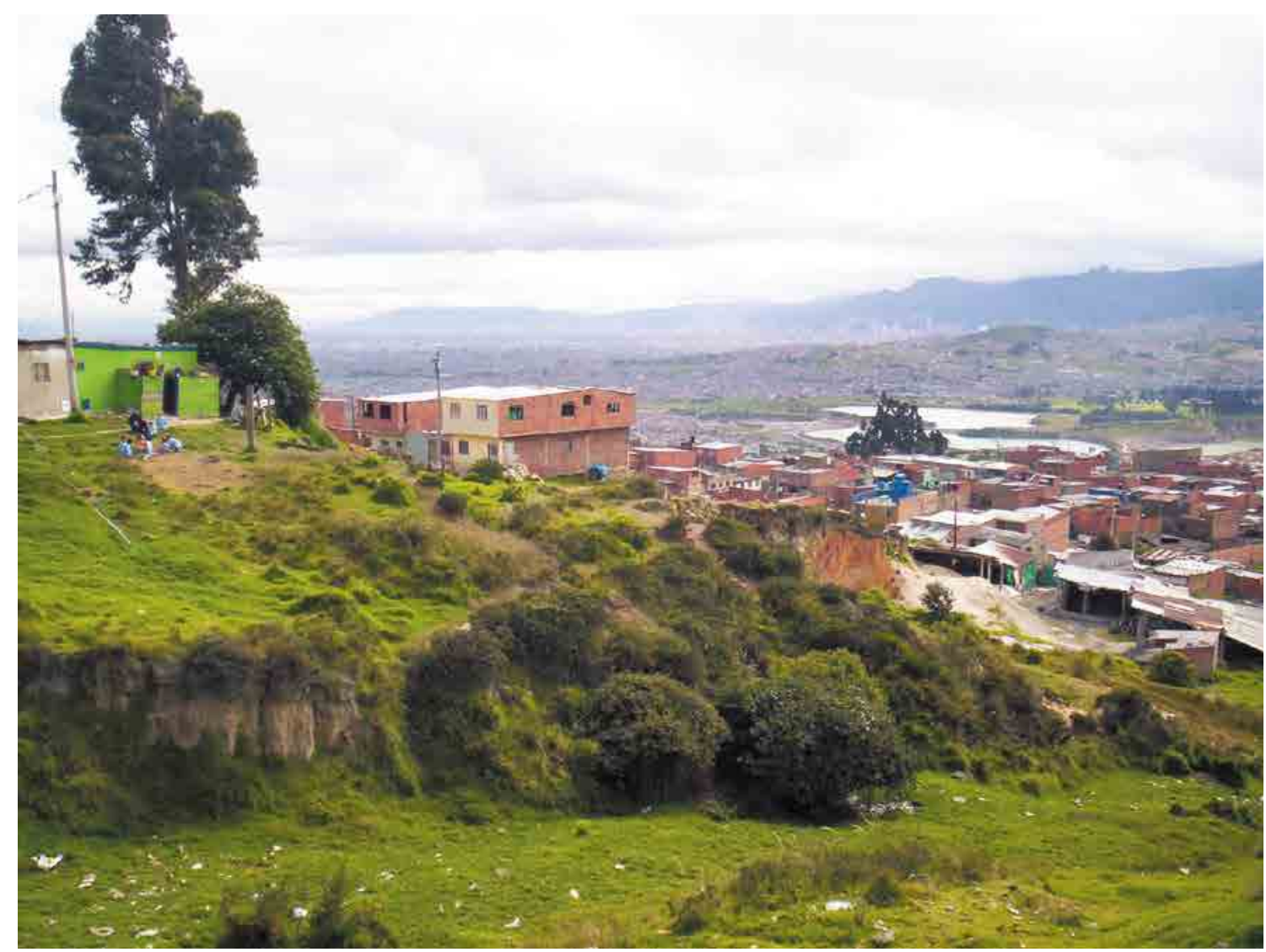

Figura 5 La ausencia de articulación entre el equipamiento y la ciudad. Sector de Ciudad Bolívar - Bogotá. Fuente: Fotografía de Jairo Ovalle (2006).

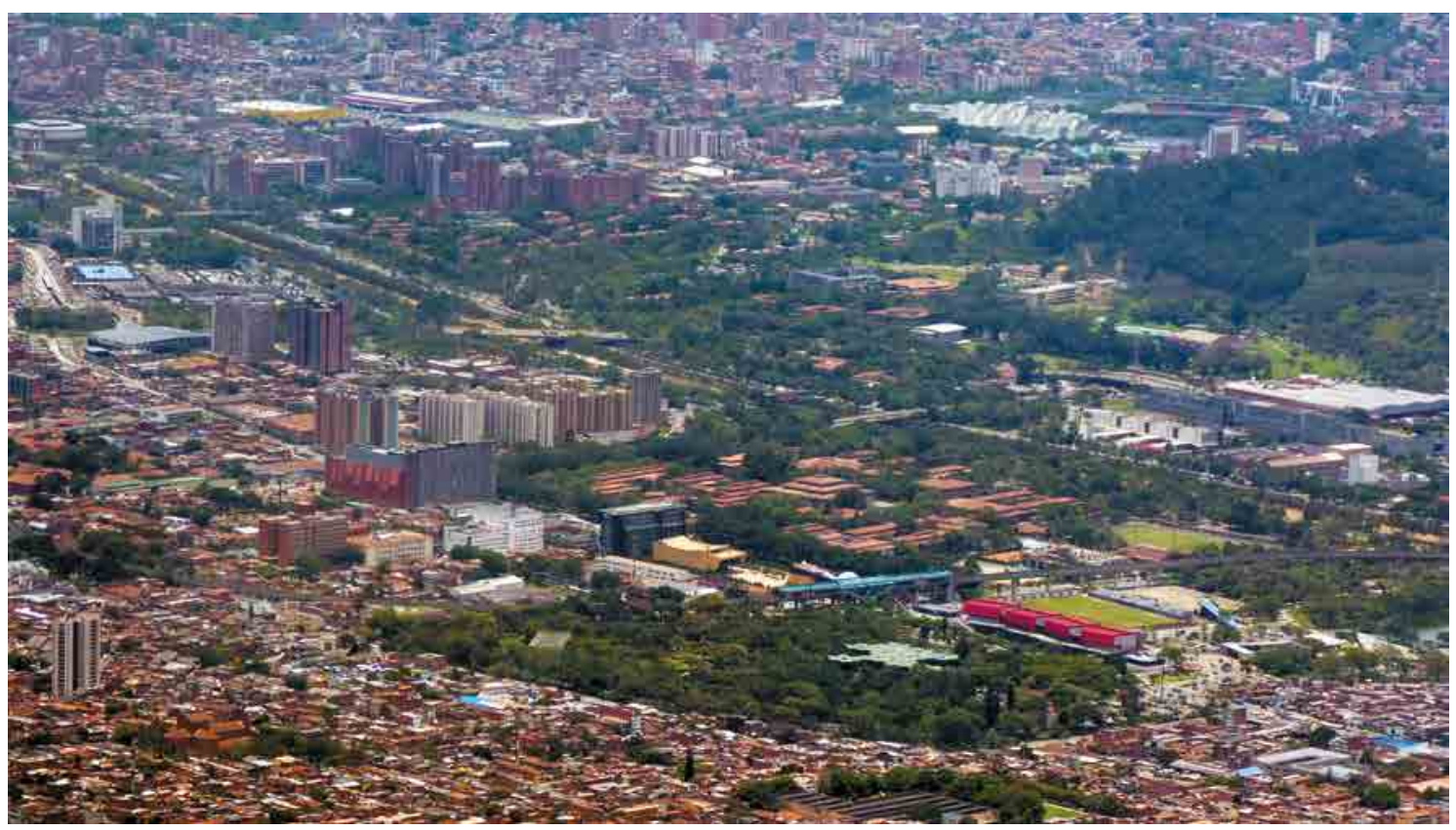

Figura 6 El papel del equipamiento en la construcción de ciudad (Medellín). Fuente: Fotografía de Jairo Ovalle (2012). 
carácter estratégico, sistemático e interdependiente sobre la denominada piel de la ciudad ${ }^{14}$, otorgándole importancia ya no solo al objeto arquitectónico, sino a la serie de intervenciones en el espacio público, fundamentales en la activación de los equipamientos a gran escala ${ }^{15}$.

Sobre esta construcción de una red se trata el modelo de "mecanismos urbanos" (Friedman, 2006), que representa la interacción de la red urbana (calles, vías, etc.) y de la efectiva atracción ejercida sobre sus habitantes a través de la importancia emocional y económica de los distintos puntos de la red.

En este marco, el Sistema Integral de Equipamientos Comunitarios 1100, ubicado en el municipio Libertador en la ciudad de Caracas, consiste en una política de reordenamiento del tejido físico y social de una comunidad que hace parte de una estructura geográfica habitable del Parque Nacional Waraira Repano. Estas intervenciones agrupan equipamientos de múltiples actividades colectivas, mejoramiento de vivienda e infraestructura de servicios, como parte de una operación de activación sistemática, en la cual las comunidades organizadas como asamblea de ciudadanos en su diseño y autoconstrucción son apoyadas a nivel humano, económico, político y social máximo ${ }^{16}$

Una operación catalizadora de una comunidad requiere, en términos de Maturana, de la apropiación de la ciudadanía, el sentido de proximidad con la ciudad y el afecto que construye el tejido social, que "constituye el dominio de conductas donde se da la operacionalidad de la aceptación del otro como un legítimo otro en la convivencia, y es ese modo de convivencia lo que connotamos cuando hablamos de lo social. Por esto digo que el amor es la emoción que funda lo social" (2013: 24).

\section{El papel del espacio público}

El último de los actores está relacionado con el espacio público, como un hecho que trasciende el sentido de espacio de libre circulación, hacia la generación de espacios considerados ambientes de diálogo con diferentes capas de privacidad en las formas de relacionar las personas, desde lo público, semipúblico y privado.

Una serie de transiciones amortiguan las diferentes escalas entre la ciudad y el equipamiento. Si bien esto implica la definición geométrica y morfológica del espacio libre (Krier, 1993), lo experimental del espacio urbano y la exaltación de la transición hacia el equipamiento considera que en dicho espacio es donde se genera la comunidad (Díaz y Marroquín, 2016).
Para que eso ocurra, se debe cumplir con tres características. La primera es la "atractividad" y su capacidad de estimular esa condición en el usuario. La segunda hace referencia al dinamismo configurado generalmente por un programa de usos abiertos, complementario a la ciudadanía. Por último, el confort, porque es innegable que en las periferias urbanas es necesario superar la noción de funcionalista de los elementos que allí se disponen, en la búsqueda de intervenciones que sean capaces de convocar a sus habitantes y retener en múltiples actividades, donde los elementos del espacio público, espacios, arborización generan una sensación de comodidad adicional a la utilidad (Iribas, 2011:19).

Tomar a Medellín como referente es reconocer el proceso de transformación física y social, donde el proyecto político traza la hoja de ruta hacia el mejoramiento de la calidad de vida de sus habitantes para articular todos los actores y gestores en torno a las intervenciones ${ }^{17}$. Para Carlos Mario Rodríguez ${ }^{18}$ (2010), los equipamientos en las operaciones en los barrios de ocupación espontánea cumplieron un papel trascendental, al pasar de garantizar una cobertura de necesidades funcionales, a construir un referente que enriquece el paisaje urbano y los articula con la ciudad, eliminando la noción de edificio objeto, aislado o desarticulado del contexto urbano, como una forma de re significación de la ciudadanía desde la consolidación de una red de servicios a lo largo del territorio de la ciudad. (Figura 6)

Concretamente, el caso de las Unidades de Vida Articuladas - Tanques de EPM (UVA) ${ }^{19}$ se trata de una serie de proyectos desarrollados en la infraestructura de abastecimiento de agua potable para la ciudad de Medellín. Estas infraestructuras, si bien consiguen el objetivo de llevar un servicio de agua de calidad a los habitantes, generaban un impacto negativo al no relacionarse con los entornos. Entre 1910 y 1950, se construyeron en la periferia del Valle de Aburrá 144 tanques que se convirtieron en lugares subutilizados, al ser absorbidos por los acelerados procesos de crecimiento urbano sobre las laderas, en su mayoría de forma precaria.

Para Horacio Valencia ${ }^{20}$, al tomar como punto de partida la comprensión de la ciudad desde la prestación del servicio de alumbrado, descubrieron de qué forma se estaba prestando el servicio y, a su vez, la relación entre un mal servicio, con factores de inseguridad y problemas con las comunidades. Así, advirtieron que en la ciudad existían zonas oscuras asociadas a territorios privados sobre los barrios, debido a tres factores principales: altas pendientes, zonas de rondas de quebradas y los tanques de abastecimiento ${ }^{21}$. Surge entonces la posibilidad de incorporar 32 tanques a los tejidos de los barrios y cambiar la concepción y el significado de la infraestructura en la construcción del territorio. Como respuesta a la carencia de espacios públicos, fueron derribados los muros de los tanques, acabando con fronteras y barreras, planteando áreas para el encuentro ciudadano. (Figura 7)

[14] "The Urban Skin" of the "epidermis", understood "as a rich, complex, and enormously influential membrene" that constitues a system in itself". cita del autor haciendo referencia al texto A Matter of Things de Manuel de Solá-Morales, publicado en 2008.

[15] El texto original de Jesús Hernández, Public Space Acupuncture, publicado en la introducción del libro "Public Space Acupuncture Strategies and Interventions for Activating City Life", de Helena Casanova y Jesús Hernández, publicado por Actar en 2014.

[16] Proyecto de las firmas AGA y PICO, ganador en la Bienal Iberoameicana de Quito 2016 en la categoría de Diseño Urbano y Arquitectura del Paisaje.

[17] Sobre estos procesos de desarrollo de equipamientos en ciudades como Bogotá y Medellín, véase artículos como "Los equipamientos urbanos como instrumentos para la construcción de ciudad y ciudadanía" de Ángela María Franco y Sandra Karime Zabala, publicado en DEARQ Revista de Arquitectura 2012 .

[18] Arquitecto que se desempeñó como Gerente de Diseño Urbano de la Empresa Municipal de Desarrollo Urbano entre los años 2004 2009

[19] Proyecto presentado en la Bienal de Venecia 2016, "Tanques de agua como parques públicos

20] Director del Departamento de Intervenciones Sostenibles de Empresas Públicas de Medellín. 


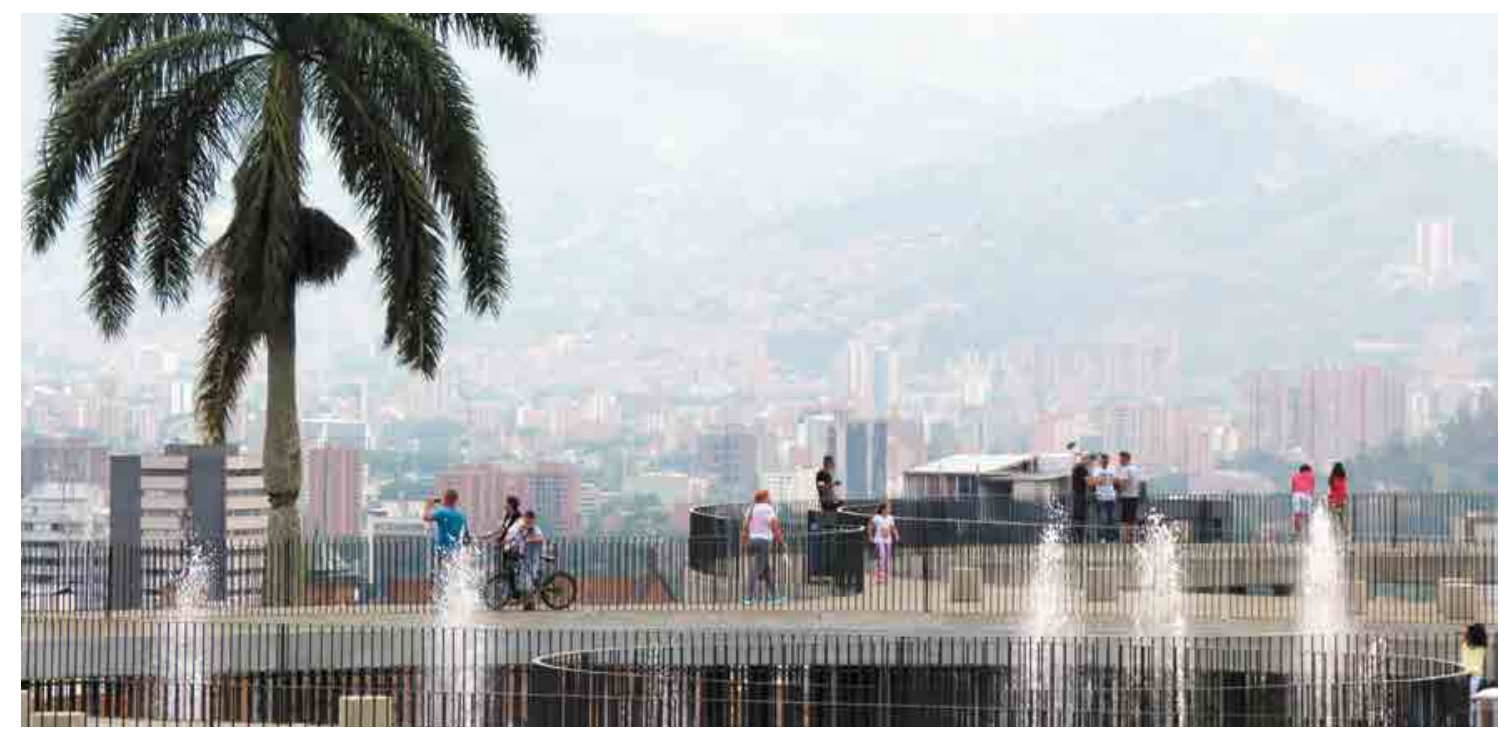

Figura 7 Unidad de Vida Articulada - Tanque La Imaginación (Medellín). Fuente: Fotografía de Jairo Ovalle (2016).

\section{La mirada sistemática sobre un repertorio}

Visto en conjunto, una sumatoria de actuaciones sobre un territorio permitiría el desarrollo de un proyecto que reconstruya los lazos comunitarios de una población. Tomando en cuenta que en los sectores urbanos de borde el déficit de equipamientos es generalizado, la mirada sistémica también aplica para los procesos de planeación donde los equipamientos "al ser prestadores de servicios múltiples en diferentes escalas y localizaciones [...] deben funcionar como una red que abarque todos los puntos de la ciudad" (Franco, 2010:7).

En este punto, la mirada del entorno indaga hacia una herramienta para entender y transmitir cómo es la ciudad que vemos y cómo funciona, cómo son las relaciones entre las personas y las huellas que aún se mantienen. La estrategia del arqueólogo como la describe Carlos Martí Arís, "emplea los vestigios encontrados para formular, a partir de ellos, una hipótesis de reconstrucción de la realidad investigada" (2005:87), una actividad equivalente al redescubrimiento de un territorio, que rastrea los vestigios, desplazamientos, costumbres y todas aquellas fuentes que conectan con el lugar, en búsqueda de una idea de transformación y de proyecto, a partir de "observar, imaginar y proyectar" $\left(\right.$ ídem) ${ }^{22}$.

Es necesario entender de manera diferente las complejidades actuales para construir un mapa complejo de decisiones a partir del análisis y llegar a la articulación de todas las variables en una resolución. Para Saskia Sassen "las ciudades son sistemas complejos, pero siempre incompletos. En esa condición reposa la posibilidad de hacer [...]. Cada ciudad es distinta y también lo es cada disciplina que la estudia. Sin embargo, si se trata de un estudio de lo urbano, deberá lidiar con esos rasgos distintivos: lo incompleto, la complejidad y la posibilidad de hacer" (Hernández et al., 2014: 15-16).
En esta complejidad, el análisis de proyectos donde se consideran aisladamente los componentes esenciales de los proyectos (Leupen, 1999) transgrede el hecho descriptivo y permite observarlos de forma simultánea. El criterio de selección está relacionado con el desarrollo gráfico que ha venido desarrollando el semillero de Análisis y Proyecto, vinculado igualmente a la Facultad de Diseño de la Universidad Católica de Colombia, que tiene como premisa que parte del conocimiento está albergado en los proyectos, y es necesario hacer evidente y poner en relación piezas inesperadas con el fin de encontrar puntos de diálogo en común.

La selección de quince proyectos realizados entre 1950 y 2015 aproximadamente, permite (aunque no todos forman parte de los procesos de expansión de la ciudad) establecer criterios de relación con el entorno que llevados a condiciones abstractas, definen los puntos comunes y las constantes desde la implantación y relación con el entorno. Posterior a esto, el repertorio fue organizado en una matriz cualitativa de proyectos para hacer evidente los criterios que bajo la lupa de la implantación contribuyen a comprender formas de superar las decisiones para enriquecer la construcción del entorno y formular alternativas ante el tímido aporte que hacen los equipamientos en la construcción del borde urbano de la ciudad de Bogotá, desde los parámetros normativos.

Los criterios de revisión están agrupados en tres temáticas:

La relación con las condiciones físico naturales (relación con el terreno, relación con factores naturales).

La relación con el contexto construido (relación con el pasado, relación con edificaciones vecinas, vinculación con el contexto).

Niveles de permeabilidad (accesibilidad, relación entre lo público y lo privado). 


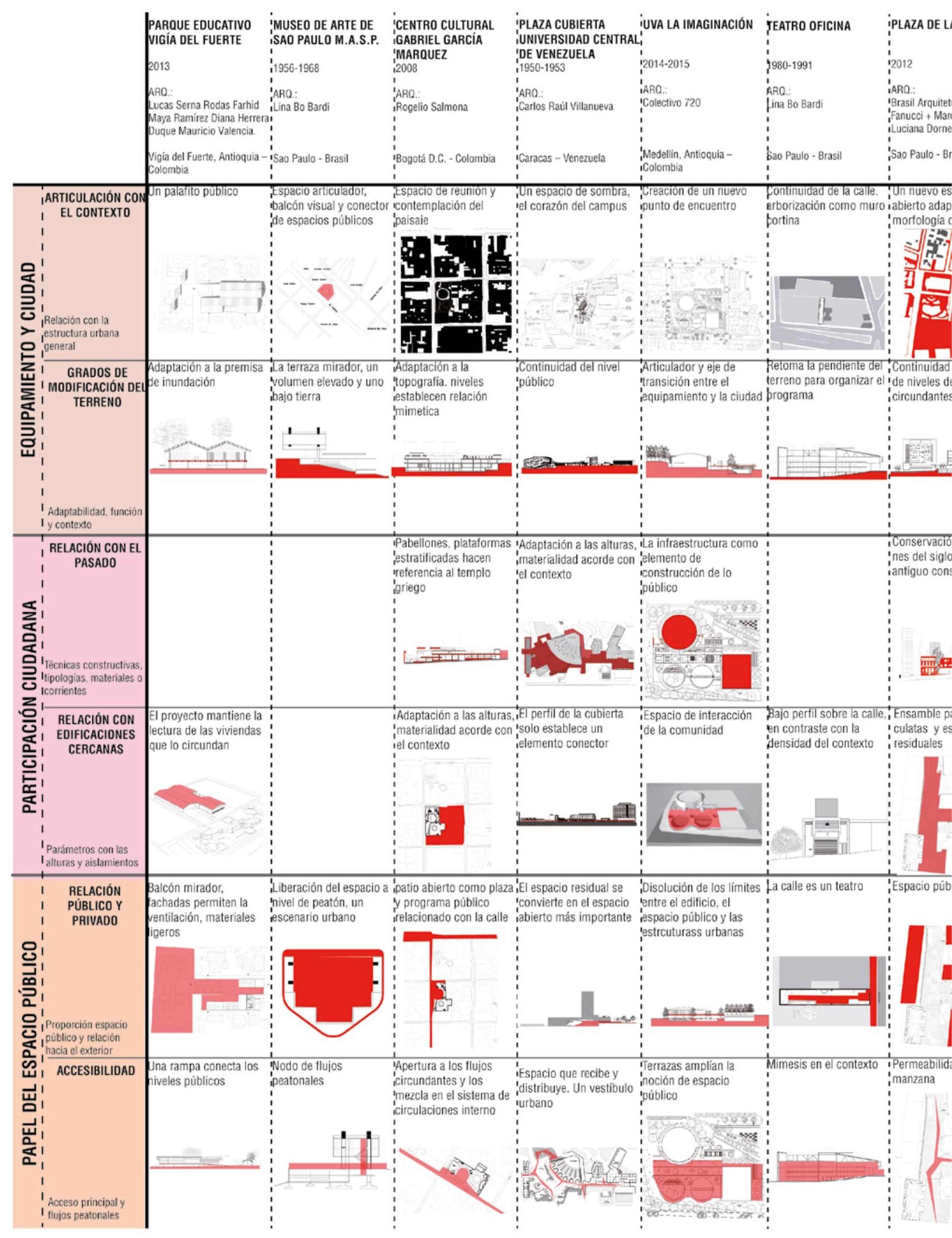

Tabla 1 Criterios de implantación y relación con el contexto de equipamientos. Fuente: Elaboración de Luisa Fernanda Torres Turner (integrante de semillero de investigación en Análisis y Proyecto, Facultad de Diseño de la Universidad Católica de Colombia) y Jairo Ovalle (2017). 


\begin{tabular}{|c|c|c|c|c|c|c|c|}
\hline $\begin{array}{l}\text { Francisco } \\
\text { Ferraz + }\end{array}$ & $\begin{array}{l}\text { ORQUIDEORAMA } \\
\text { 2005-2006 } \\
\text { 'ARQ: } \\
\text { 'Plan B Arquitectos + JPCR } \\
\text { Arquitectos } \\
\text { Medellin, Antioquia - } \\
\text { Colombia }\end{array}$ & $\begin{array}{l}\text { 'MUSEO DE ARTE DEL } \\
\text { 'BANCO DE LA } \\
\text { 'REPUEBLICA } \\
\text { '2004 } \\
\text { ARO.: } \\
\text { Enrique Triana y Juan Carlos } \\
\text { Rojas } \\
\text { Bogotá D.C. - Colombia } \\
\text { ing }\end{array}$ & $\begin{array}{l}\text { PLAZA DO PATRIARCA } \\
\text { 1992-2000 } \\
\text { ARQ.: } \\
\text { Paulo Mendes Da Rocha } \\
\text { Sao Paulo - Brasil } \\
\text { Sal }\end{array}$ & $\begin{array}{l}\text { IFAU } \\
\text { 'UNIVERSIDAD DE SAO } \\
\text { IPAULO } \\
\text { '1961-1969 } \\
\text { 'ARQ.: } \\
\text { 'João Batista Vilanova } \\
\text { IArtigas e Carlos Cascaldi } \\
\text { 'Sao Paulo - Brasil } \\
\text { is }\end{array}$ & $\begin{array}{l}\text { 'COLEGIO SANTO } \\
\text { 'DOMINGO SAVIO } \\
\text { 2008 } \\
\text { 'ARQ.: } \\
\text { Obranegra Arquitectos } \\
\vdots \\
\text { IMedellin, Antioquia - } \\
\text { Colombia }\end{array}$ & $\begin{array}{l}\text { IM U B E } \\
\vdots \\
1995 \\
\text { ARQ.: } \\
\text { Paulo Mendes Da Rocha } \\
\vdots \\
\text { Sao Paulo - Brasil } \\
\text { ing }\end{array}$ & $\begin{array}{l}\text { ARENA DO MORRO } \\
\text { '2014 } \\
\text { ARQ: } \\
\text { Herzog \& De Meuron } \\
\text { 'Río Grande do Norte - Brasil } \\
\text { A }\end{array}$ \\
\hline nanzana & $\begin{array}{l}\text { Ta plaza cubierta como } \\
\text { escenario urbano } \\
\vdots \\
\vdots \\
\vdots \\
\vdots\end{array}$ & 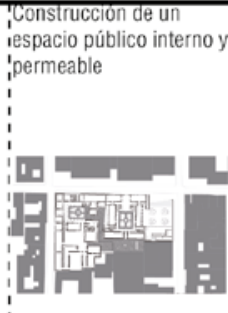 & $\begin{array}{l}\text { Modificacion de la escala } \\
\text { para establecer una } \\
\text { 'relación con la ciudad } \\
\end{array}$ & $\begin{array}{l}\text { ádu contenedor publico } \\
\text { Ide acatividades } \\
\text { 'académicas }\end{array}$ & $\begin{array}{l}\text { Disolución vertical det } \\
\text { ilos limites entre el } \\
\text { 'espacio privado y } \\
\text { ipúblico } \\
1 \\
1\end{array}$ & $\begin{array}{l}\text { 'La sombra en el jardin } \\
\text { isobre un basamento } \\
\text { 'excavado } \\
\vdots \\
\vdots \\
\vdots \\
\vdots \\
\end{array}$ & $\begin{array}{l}\text { Articulador urbanoy y } \\
\text { irelación lejana con el } \\
\text { 'paisaie maritimo } \\
\end{array}$ \\
\hline $\begin{array}{l}\text { onexión } \\
\text { s calles }\end{array}$ & $\begin{array}{l}\text { Continuidad y conexión } \\
\text { de niveles de las calles } \\
\text { circundantes } \\
\text { a... }\end{array}$ & 'Enterrado en el suelo & $\begin{array}{l}\text { La plaza determina los } \\
\text { niveles } \\
\text { and }\end{array}$ & $\begin{array}{l}\text { Programa enterrado, } \\
\text { 'eve cambio como } \\
\text { 'transición }\end{array}$ & $\begin{array}{l}\text { framo escalonado, y } \\
\text { otro como plataforma } \\
\text { balcón } \\
\vdots \\
1\end{array}$ & 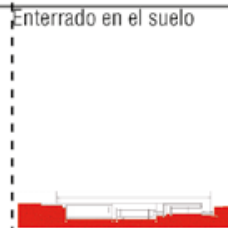 & $\begin{array}{l}\text { Adaptación a la } \\
\text { pendiente del terreno } \\
1 \\
1\end{array}$ \\
\hline & & & 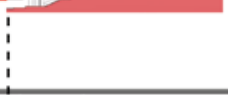 & & : & $\begin{array}{l}1 \\
1 \\
1 \\
1 \\
1\end{array}$ & 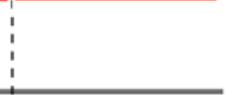 \\
\hline $\begin{array}{l}\text { dificacio- } \\
X, \\
\text { vatorio }\end{array}$ & $\begin{array}{l}\text { In lugar adaptado a la } \\
\text { 'tradición como nuevo } \\
\text { iescenario } \\
\text { ' } \\
1 \\
1 \\
1\end{array}$ & $\begin{array}{l}\text { IUn patio interior } \\
\text { recupera la tipología } \\
\text { ioriginal de manzana. } \\
\\
\end{array}$ & 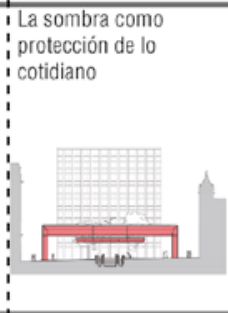 & : & ' & $\begin{array}{c}1 \\
\vdots \\
1 \\
1 \\
1 \\
1 \\
1 \\
1 \\
1 \\
1 \\
\end{array}$ & : \\
\hline $\begin{array}{l}\text { evitar } \\
\text { ioss }\end{array}$ & & 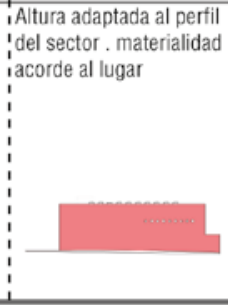 & $\begin{array}{l}\text { Altura adaptada al perfil } \\
\text { del sector . materialidad } \\
\text { acorde al lugar }\end{array}$ & & $\begin{array}{c}1 \\
\vdots \\
1 \\
1 \\
1 \\
1 \\
1 \\
1 \\
1 \\
\end{array}$ & 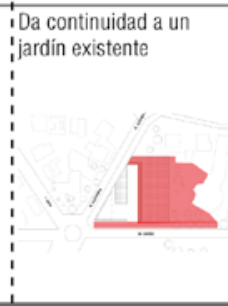 & $\begin{array}{l}\text { i Continuidad con el pe } \\
\text { 'horizontal del sector } \\
\text { ing }\end{array}$ \\
\hline ineal & $\begin{array}{l}\text { Espacio de encuentro, } \\
\text { abierto, eleimina la } \\
\text { Inoción de edificio }\end{array}$ & $\begin{array}{l}\text { 'Patio abierto como plaza } \\
\text { 'y programa público } \\
\text { ínelacionado con la calle } \\
\text { '. }\end{array}$ & $\begin{array}{l}\text { Una estructura de } \\
\text { 'sombra, articulada } \\
\text { estructura urbana } \\
\text { and }\end{array}$ & $\begin{array}{l}\text { Diferentes escalas de } \\
\text { relación entre el edificio } \\
\text { 'y el campus } \\
1 \\
\end{array}$ & $\begin{array}{l}\text { La plaza mirador como } \\
\text { 'principal acontecimien- } \\
\text { ito, un nuevo lugar en el } \\
\text { 'barrio } \\
\\
\end{array}$ & 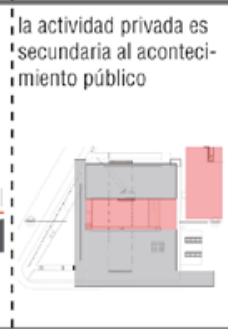 & $\begin{array}{l}\text { 'Permeabilidad acentuada } \\
\text { ipor la celosía y la } \\
\text { 'cubierta a dos aguas }\end{array}$ \\
\hline & $\begin{array}{l}\text { Espacio de distribuci } \\
\text { nodo urbano } \\
1 \\
100\end{array}$ & Secuencia de espacios & $\begin{array}{l}\text { Patio abierto como plaza } \\
\text { iy programa público } \\
\text { 'relacionado con la calle }\end{array}$ & $\begin{array}{l}\text { âfluidez en el sistema de } \\
\text { circulación articulado } \\
\text { con el campus }\end{array}$ & $\begin{array}{l}\text { 'Separación de flujos, } \\
\text { iprivados y públicos para } \\
\text { 'el funcionamiento } \\
\text { 'simultáneo }\end{array}$ & $\begin{array}{l}\text { Permeabilidad al jardín, } \\
\text { icontrol al interior de la } \\
\text { 'edificación }\end{array}$ & $\begin{array}{l}\text { IMarcada direccionalidad } \\
\text { 'dada por las superficies } \\
\text { icurvas } \\
\text { ing }\end{array}$ \\
\hline
\end{tabular}




\section{CONCLUSIONES}

Este texto plantea la identificación de tres parámetros básicos para el desarrollo de los equipamientos en los contextos de borde urbano, en el marco de un desarrollo metodológico para intervenir el borde urbano suroriental de la ciudad de Bogotá. Sus definiciones y alcances desde los aspectos morfológicos, la participación de la comunidad y su relación con el espacio público, están enfocados en la superación de la condición deficitaria y precaria que, por lo general, prevalece en las políticas de intervención y cuestiona las determinantes que desde el proyecto arquitectónico y la planeación deben ser tomadas en cuenta a la hora de intervenir estos contextos.

En este sentido, recuperar la discusión acerca del rol estructurante del equipamiento en la construcción de la ciudad, trasciende el hecho del objeto arquitectónico a la luz de las intervenciones desarticuladas que se han llevado a cabo en el sector de Yomasa, que al no ser ajenas a las problemáticas de la ciudad latinoamericana, permiten una revisión desde lo local, como también establecer una forma de aproximación que potencialice las condiciones urbanas de esta clase de proyectos.

A nivel de la población, el rol que adquieren los habitantes en la modelación de las políticas que rigen la construcción de este tipo de territorios es fundamental, en la medida que permite una aproximación más cercana a los problemas particulares de las comunidades, dejando de lado la condición genérica del habitante urbano a cambio de un agente de participación directa en la toma de decisiones de la construcción del territorio, donde el equipamiento es insertado.

Para terminar, y en relación a los puntos anteriores, el desarrollo de una matriz basada en principios básicos de análisis retomados de la escuela de Delft, establece la continuidad en la construcción de un insumo para aproximar proyectos distantes. Se trata de un documento abierto que permite agregar o retirar piezas, explorando alternativas para el desarrollo de espacios y lugares que superan la satisfacción de las necesidades básicas, en busca de parámetros arquitectónicos, que modifican nuestra aproximación desde la disciplina pero también que abren un espacio a lo común, aquellos lazos comunitarios manifestados en el espacio urbano. 


\section{REFERENCIAS BIBLIOGRÁFICAS}

ALEXIOU, loannis. El sistema de equipamientos, sistema estructurante de la ciudad Metropolitana, Revista Escala, 2004, n 196, pp. 5-14.

AMAYA, Luis; ALEXIEU, loannis; SUÁREZ, Gabriel; SERNA, David y RUEDA, Liliana. Diálogos con escala. Soportes urbanos, Revista Escala, 2004, n 196, pp. 15-19

CASANOVA, Helena y HERNÁNDEZ, Jesús. Public Space Acupuncture, Strategies and Interventions for Activating City Life. Nueva York: Editorial Actar, 2014.

CENTRO DE INVESTIGACIONES DE LA FACULTAD DE DISEÑO, CIFAR. El impacto de los equipamientos colectivos en los procesos de rehabilitación urbana - caso de estudio borde urbano de Bogotá - Gran Yomasa. Documento de formulación inédito de la convocatoria interna de proyectos de investigación 2016, Universidad Católica de Colombia, 2015.

CRUZ, Valdemar. Álvaro Siza, conversaciones con Valdemar Cruz. Barcelona: Editorial Gustavo Gili, 2007.

DÍAZ, Myriam y MARROQUÍN, Julio. Las relaciones entre la movilidad urbana y el espacio público. Transmilenio en Bogotá, Revista de Arquitectura, 2016, vol., $18, n^{\circ} 1,126-139$.

FRANCO, Ángela. Ciudad y Equipamientos, relaciones para el desarrollo social, Revista Escala, 2010, n² 219, pp.7-12.

FRANCO, Ángela y ZABALA. Sandra. Los equipamientos urbanos como instrumentos para la construcción de ciudad y ciudadanía, Revista DEARQ, 2012, n 11, pp. 10-21.

FRIEDMAN, Yona. Pro Domo. Barcelona: Editorial Actar, 2006.

HERNÁNDEZ, Alejandro; SASSEN, Saskia; DELGADO, Manuel; PALLASMAA, Juhani; MÜLLER, Willy; HEREU, Jordi; MOCKUS, Antanas; SÁNCHEZ, Osvaldo; VILLORO, Juan y LIDA, David. Habla Ciudad. México D.F: Arquine, 2014.

IRIBAS, José Miguel. Elogio del Encuentro, espacio público, espacio ciudadano, Revista Arquitectura Viva, 2011, n’ 136 Escenarios Urbanos, pp. 19.

KRIER, Rob. Architecture and urban design. University of Michigan: Academy Editions, 1993.

LYNCH, Kevin. La imagen de la ciudad. Buenos Aires: Ediciones Infinito, 1970.

LEUPEN, Bernard; GRAFE, Christoph; KÖRNIG, Nicola; LAMPE, Mark y DE ZEEUW, Peter. Proyecto y análisis. Evolución de los principios en Arquitectura. Barcelona: Editorial Gustavo Gili, 1999.

MARTÍ ARÍS, Carlos. La cimbra y el arco. Barcelona: Fundación Caja de Arquitectos, 2005.

MATURANA, Humberto. Emociones y lenguaje en educación y política. Santiago: JC Sáez Editor, 2013.
MORIN, Edgar. El pensamiento complejo. Barcelona: Editorial Gedisa, 1998.

RODRÍGUEZ, Carlos Mario. Equipamientos. Ordenar la ciudad, Revista Escala, 2010, n²19, pp.13-16.

ROGERS, Ernesto; SERT, Josep y TYRWHITT, Jacqueline. El corazón de la ciudad, por una vida más humana de la comunidad. Barcelona: Hoepli, S.L., 1955.

ROSSI, Aldo. La arquitectura de la ciudad. Barcelona: Editorial Gustavo Gili, 1971.

RUBIO, Rodrigo y CASTELLANOS, Doris. Ciudades urgentes: Intervenciones en áreas urbanas de crecimiento rápido. Bogotá: Departamento de Arquitectura, Universidad de los Andes, 2006

URREA, Tatiana; del CASTILLO, Juan Carlos y MONTAÑA, Jimena. Usme: Historia de un territorio. Bogotá: Alcaldía Mayor de Bogotá - Metrovivienda, 2011.

VERGARA, Alfonso y DE LAS RIVAS, Juan Luis. Territorios inteligentes. $1^{\text {a }}$ edición. Madrid: Fundación Metrópoli, 2014 